at least one mental disorder or neuropsychiatric manifestation, the most frequently reported was depression.

\section{P148 A STUDY OF DISEASE PROFILE OF ADULT AND JUVENILE LUPUS PATIENTS AT DISEASE ONSET AT TERTIARY CARE CENTRE OF NORTHERN INDIA}

${ }^{1}$ Neeraj Jain, 'Lalit Duggal, ${ }^{2}$ Sujata Sawhney, ${ }^{2}$ Manjari Agarawal, 'Jeet Kumar Patel, ${ }^{1}$ Mayank Gupta. ${ }^{1}$ Dept. of Rheumatology, Sir Ganga Ram Hospital, New Delhi; ${ }^{2}$ Dept. of Pediatric Rheumatology, Sir Ganga Ram Hospital, New Delhi, India

\subsection{6/lupus-2020-eurolupus. 191}

Background Systemic Lupus Erythematosus (SLE) is a chronic autoimmune disease. It may affect adults and paediatric patients and may have different organ involvement at onset of disease.

Objectives To study disease profile of 100 adult and paediatric SLE patients at disease onset to find any significant difference in organ involvement in both cohorts.

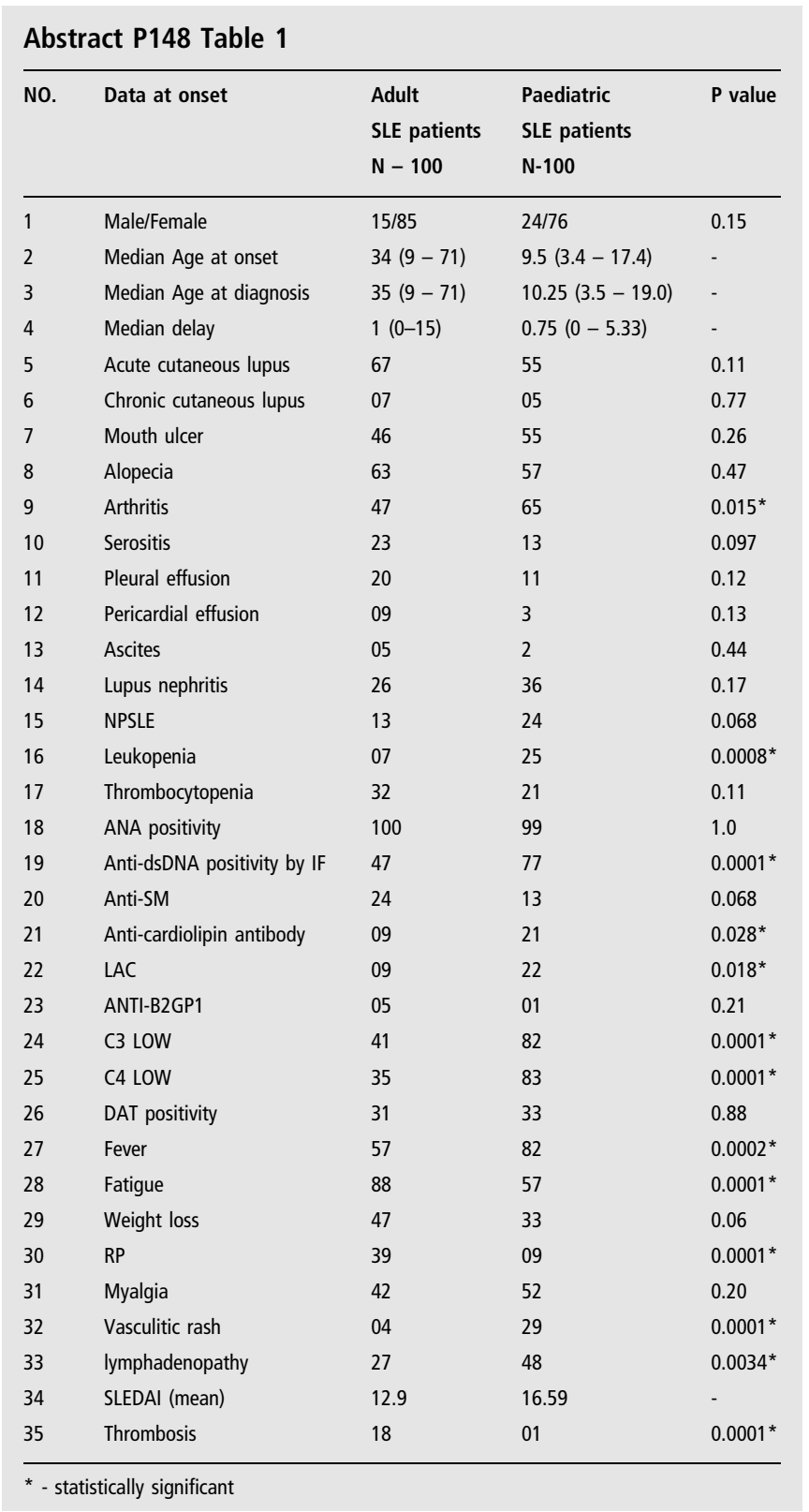

Method 100 adult and 100 paediatric patients who classified SLE-SLICC criteria were recruited in study after taking consent at tertiary care centre in northern India. Demographic data and clinical profiles were recorded. Fisher's test was used to find $\mathrm{p}$ value and $\mathrm{p}$ value less than 0.05 was taken as statistical significance.

Results Out of 100 patients, females were $85 \%$ in adult cohort Vs $76 \%$ in paediatric cohort. Median delay in diagnosis was more with adult than paediatric cases. Lupus nephritis, mouth ulcers, NPSLE was common in paediatric SLE patients. In adult cohort, there was significant association for fatigue, $\mathrm{RP}$, and thrombosis.

Conclusion Major organ involvement was frequent in adult patients while arthritis, leukopenia, low complements, more positivity of anti-dsDNA, APLA antibodies positivity were significantly common in paediatric cases.

\section{P149 ASSOCIATION BETWEEN GEOGRAPHIC AND CLIMATOLOGICAL CONDITIONS AND CUTANEOUS MANIFESTATIONS IN LUPUS PATIENTS FROM THE SPANISH RHEUMATOLOGY SOCIETY LUPUS REGISTRY COHORT}

${ }^{1}$ Raúl Menor-Almagro, ${ }^{2}$ Alejandro Muñoz, ${ }^{3}$ ñigo Rúa-Figueroa, ${ }^{4}$ María Auxiliadora MartínMartínez, ${ }^{5}$ Alejandro Olivé, ${ }^{6}$ María Galindo, ${ }^{7}$ Jaime Calvo-Alén, ${ }^{8}$ Antonio Fernández-Nebro, ${ }^{9}$ Javier López-Longo, ${ }^{10}$ Mariano Andrés, ${ }^{11}$ Eva Tomero, ${ }^{12}$ Esther Uriarte, ${ }^{13}$ Mercedes Freire, ${ }^{14}$ Javier Narváez, ${ }^{15}$ José María Pego-Reigosa, on behalf of RELES-SER. ${ }^{1}$ Rheumatology, Hospital Jerez, Jerez; ${ }^{2}$ Rheumatology, Hospital Virgen del Rocío, Sevilla; ${ }^{3}$ Rheumatology, Hospital Doctor Negrín, Las Palmas GC; ${ }^{4}$ Research Unit, Sociedad Española de Reumatología, Madrid; ${ }^{5}$ Rheumatology, Hospital Germans Trias i Pujol, Badalona; ${ }^{6}$ Rheumatology, Hospital 12 de Octubre, Madrid; ${ }^{7}$ Rheumatology, Hospital Txagorritxu, Vitoria; ${ }^{8}$ Rheumatology, Hospital Regional Universitario Málaga, Málaga; ${ }^{9}$ Rheumatology, Hospital Gregorio Marañón, Madrid; ${ }^{10}$ Rheumatology, Hospital Alicante, Alicante; ${ }^{11}$ Rheumatology, Hospital La Princesa, Madrid; ${ }^{12}$ Rheumatology, Hospital Donostia, Donostia; ${ }^{13}$ Rheumatology, Hospital Juan Canalejo, A Coruña; ${ }^{14}$ Rheumathology, Hospital Bellvitge, Barcelona; ${ }^{15}$ Hospital Meixoeiro, Vigo, Spain

\subsection{6/lupus-2020-eurolupus. 192}

Background/Purpose Ultraviolet radiations act by modifying DNA in sun-exposed skin of lupus patients. We develop a study to analyze the association between climatological conditions and cutaneous manifestations in systemic lupus erythematosus (SLE).

Methods Patients data from Spanish Rheumatology Society Lupus Registry (RELESSER) cohort were retrospectively analyzed for presence of cutaneous lesions (alopecia, photosensitivity, malar rash, discoid lesions, oral ulcers and subacute lesions). We included patients who were assessed in rheumatology services from January 2011 to December 2012. Data of climatological conditions throughout the Spanish geography were provided by the Spanish Meteorological Agency.

Results A total of 2919 patients were included, 87.3\% female. Others biological and clinical data are showed in table 1 . In the multivariable model, positive associations were observed between coastal regions OR $1.470 \quad(95 \%$ CI:1.080-2.001 $\mathrm{p}=0,014)$, anti-DNA OR $1.806 \quad(95 \%$ CI:1.276-2.556, $\mathrm{p}=0.001)$, antiphospholipids antibodies OR $1.428 \quad(95 \%$ CI:1.093-1.864 p=0.009), serositis OR 1.557 (95\% CI:1.181$2.053 \mathrm{p}=0.002)$ and arthritis OR 1.804 (95\% CI:1.258-2.587 $\mathrm{p}=0,001)$. Negative associations were observed between females OR 0.412 (95\% CI:0.284-0.599, p=0.000) and antimalarial drugs OR 0.469 (95\% CI:0.327-0.671, p=0.000).

Conclusion Although the influence of global and ultraviolet radiations on the development of cutaneous lesions in SLE 